\title{
EMISSION OF ACCRETION DISKS
}

\author{
B. CZERNY* \\ Copernicus Astronomical Center, Bartycka 18, 00-716 Warsaw, Poland
}

\begin{abstract}
Accretion disks surrounding massive black hole is an attractive scenario of nuclear activity. A number of arguments support it although there is no unquestionable proof of the existence of accretion disks in active galactic nuclei. Meaningful comparison of the disk model prediction with the data can only be made if emission of accretion disks is calculated taking into account the existence of optically thin parts responsible for the emission of $x$-ray radiation. Nonlocal reprocessing phenomena have to be also included. Since we have no real understanding of the viscous processes operating in accretion disks some ad hoc parameterization of these processes has to be used and its applicability should be checked by broad band comparison of predictions for continuum emission and spectral features with available data.
\end{abstract}

Key words: accretion disks, thermal emission, optical/uv spectra, $x$-ray spectra

\section{Introduction}

The nature of nuclear activity in galaxies remains a mystery although a huge amount of data have already been accumulated over the energy range from radio to gamma. This is the result of unquestionable complexity of the phenomenon.

It is widely believed that the ultimate source of energy is accretion onto supermassive black holes, as suggested by Zeldovich \& Novikov (1964) and Salpeter (1964). The clear advantage of such a scenario (Rees 1984) is the compactness of the activity center, its efficiency and stability, and the possibility of multiple reactivation of the nucleus by the fresh supply of matter. However, attempts are made to explain all the nuclear activity through starburst phenomenon (e.g. Terlevich et. al. 1992).

The overall character of accretion is not so clearly specified as in the case of $\mathbf{x}$ ray binaries (e.g. Frank, King and Raine 1985). The accreting gas in active galactic nuclei most probably comes from the inner $\sim 10 \mathrm{kpc}$ losing its angular momentum through gravitational interaction (Schlosman, Begelman \& Frank 1990) but an inflow more resembling the cooling flow may also be a possibility (see Heckman 1992).

The overall view of an active nucleus is determined not only by the energy release close to the center of activity but also by the circumnuclear processes: collimation and acceleration of jets and modification of outgoing radiation by scattering and absorption by dust and ionized medium surrounding an nucleus at $0.01-100 \mathrm{pc}$. Because of anisotropic character of these phenomena, the result strongly depends on the inclination angle. Unification schemes suggested recently (see Barthel 1992), although not perfect (Antonucci, this volume), help to disantangle the central phenomenon from these secondary effects.

* Also Observatoire de Paris-Meudon

261

T. J.-L. Courvoisier and A. Blecha: Multi-Wavelength Continuum Emission of AGN, 261-270.

(C) 1994 IAU. Printed in the Netherlands. 
The AGN spectra and observed variability clearly indicate the coexistence of hot and cold gas within the inner $\sim 1$ pc (see Mushotzky, Done \& Pounds 1993). However, this is possible within two basically different scenarios. According to the first one, most of the gravitational energy is released within hot accreting medium in a form of $x$-ray emission, and the cold blobs coexist with the hot phase (as a result of thermal instabilities and confinement by magnetic field) reprocessing a fraction of the primary radiation. According to the second one, accreting gas is relatively cold, disk like and optically thick, and only in some (innermost ?) part of the flow, due to thermal instabilities, the temperature rises and a fraction of energy is released in a form of $x$-rays.

Some other, more complex, possibilities can be imagined as well (e.g. Fabian \& George 1990) but they combine the properties of the two cases described above. In this review I discuss the second scenario, with particular emphasis on the observational verification of the models through the prediction of their emission spectra.

\section{Disk accretion}

Accretion disks surrounding massive black holes were suggested as a plausible scenario of nuclear activity by Lynden-Bell (1969). The early papers of Shakura and Sunyaev (1973) and Novikov and Thorne (1973) formed a theoretical basis (see Pringle 1981). The contribution of the disk to the AGN spectrum was expected in the uv band and it was identified with observed blue bump component by Shields (1978). However, if we want to explain the overall activity of a nucleus we have to go beyond those first simple models. Particularly, we should include:

- possible radial and vertical stratification of the disk,

- global effects of reprocessing of radiation emitted by one part of the disk by the other parts,

- time evolution.

\subsection{ARguments in Favor of Disk aCCREtion}

There are no proofs of the existence of accretion disks at the active nuclei. However, a number of arguments have been broadly discussed in a number of review articles (e.g. Collin-Souffrin 1992). To summarize them briefly, there are two basic arguments. The first is the observational one: clearly axial - not the spherical! symmetry of the nucleus, as adopted in all unification schemes (see Antonucci, this volume). A number of phenomena indicates that: (i) presence of jets and anisotropic radio emission (ii) dust/molecular torus (iii) ionization cone/ENLR (iv) $x$-ray reflected component (v) double profile component in emission lines. The second is the theoretical one: the disk accretion is highly efficient (5\% for nonrotating black hole and up to $42 \%$ for a rotating one) whilst the efficiency of spherical accretion is usually much lower (and difficult to estimate).

There are also some arguments against accretion disks (e.g. Antonucci 1992) but these arguments are related to particular disk models and cannot be dealed 
with before establishing the vertical disk structure (Malkan 1992).

\subsection{EXPECTATIONS FROM THE MODEL}

We do not expect to explain the blazar component at this stage of modelling as the formation of jet is a very complex problem in itself. Although there are attempts to describe this phenomenon in the context of accretion disks (e.g. Romanova \& Lovelas, this volume) they necessarily involve assumptions about the global properties of magnetic field within the disk and outside. In this review I concentrate on AGN which are both radio-quiet and unabsorbed by circumnuclear dust.

\subsubsection{Optical/uv continuum}

Large samples of AGN (e.g. Neugebauer et. al. 1987, Sanders et. al. 1989) clearly show the presence of big blue bump extending from optical frequencies upwards. The universal position of the minimum at $\log \nu \sim 14.5$ (or $\lambda \sim 1 \mu \mathrm{m}$ ) results from the evaporation of dust grains at the temperature about $1500 \mathrm{~K}$ so the dust contribution to the spectrum does not extend beyond near-ir (see Barvainis 1992). High frequency extension of the big bump is not well constrained. In (unobscured) Seyfert galaxies and low redshift quasars the decline of the spectrum is frequently observed in far-uv (Edelson and Malkan 1986, O'Brien, Gondalekhar and Wilson 1988) whilst in many high redshift quasars no cut-off is observed even up to $\log \nu \sim$ $15.7(\lambda \sim 650 \AA)$ (e.g. Bechtold et al. 1984, Reimers et al. 1992). The soft x-ray excesses present in 50\% - 90\% of Seyferts and quasars (Wilkes and Elvis 1987, Walter and Fink 1993) do not necessarily mean that the big blue bump extends into soft $x$-rays. In contrary, the universal shape of most of the excesses (Walter and Fink 1993) are better explained as spectral features due to reprocessing of x-rays by weakly ionized gas, e.g. accretion disk (Czerny et al., this volume). This spectral component is expected to come from optically thick (in a sense of effective optical depth) parts of accretion disk.

\subsection{2. $X$-ray continuum}

Recent data from Compton Observatory indicate that the $x$-ray continuum in radio-quiet objects does not extend beyond $\sim 100 \mathrm{keV}$ and it is predominantly thermal (see Zdziarski, this volume). This conclusion is based both on the positive detection for a few sources as well as non-detection of a number of Seyfert galaxies. This spectral component is expected from optically thin parts of accretion disk, mostly cooled by Compton scattering.

\subsubsection{Spectral features}

High resolution of $x$-ray data allows to study such features as absorption edges, emission lines (particularly $\mathrm{K}_{\alpha}$ iron line) and recombination continua as well as Compton reflection. Significant part of these features may (or should) form as the result of reprocessing of x-ray flux by the optically thick cool parts of accretion disks. Also some uv and optical low ionization emission lines may (and should) 
come from the disk (e.g. Dumont \& Collin-Souffrin 1990)

\section{Keplerian stationary local black body model}

This simplest model has been widely used to model the AGN spectra, starting from Malkan (1983). The underlying assumptions (see Pringle 1981, Frank, King and Raine 1985) reduce the problem of disk structure so significantly that the flux generation at a given disk radius $r$ is given by a simple formula and the disk spectrum is fully described by the mass of the black hole $M$, accretion rate $\dot{M}$, and the inclination angle $i$. The applicability of the model is constrained by the critical value of the accretion rate dependent on the efficiency $\eta$ of accretion: $\dot{M}_{\text {crit }}=$ $L_{E d d} / c^{2} / \eta$, as 'slim disks' effects become important when the luminosity of the disk is comparable or larger than the Eddington luminosity $L_{E d d}$ (e.g. Abramowicz et al. 1988).

Assumptions of stationarity and local emmissivity given by black body cause that the description of the spectrum neither contains nor requires any information about the disk structure.

First applications of this model were encouraging as the model gives a spectrum peaking in the uv range on $\nu F_{\nu}$ diagram. This is, however, not surprising, as any thermal emission at a fraction of the Eddington luminosity expected for a $10^{8} M_{\odot}$ black hole would give the same range of temperatures (Rees 1984). Also, closer examination reveals a number of problems: (i) the energy index in the optical/uv slope $\left(F_{\nu} \sim \nu^{-\alpha}\right)$ predicted by the model (asymptotically approaching $-1 / 3$ at long wavelengths) is too small; mean values are $\sim 0.2$ for quasars (Neugebauer et al. 1987 ) and $~ 0.5$ for Seyferts (Malkan 1988) (ii) x-ray emission is not predicted for by the model. Although a plausible scenario explains $x$-ray emission as originating in shocks developing in the gas outflowing along the symmetry axis, i.e. above the accretion disk (Henri \& Pelletier 1991), this x-ray emission is not accounted for energetically.

\section{Transfer of radiation and of angular momentum}

Any departure from local black body assumption requires:

- description of opacities,

- description of angular momentum transfer and the energy dissipation.

Inclusion of bound-free and bound-bound opacities is essential, particularly in the outer part of the disk (Hure, Collin and Pineau des Forêts, this volume). Some progress has been made along this line (e.g. Kolykhalov and Sunyaev 1984, Czerny and Elvis 1987, Ross, Fabian and Mineshige 1993) but still more improvements are required.

As for the angular momentum transfer, we have no real understanding of its mechanism. From the practical point of view, there are three possibilities:(i) angular momentum is transported locally and rather uniformly within disk (ii) the 
transport and dissipation is still local but significantly enhanced either close to the disk surface or to the equatorial plane (iii) the transport is truly global, e.g. through global magnetic field or spiral waves.

\section{Alpha viscosity disks}

Simple parameterization of the angular momentum transfer was introduced by Shakura and Sunyaev (1973). Angular momentum is transported by some anomalous viscosity and the viscous torque $\tau$ is proportional to the pressure. Two cases are widely discussed: torque proportional to the gas pressure and torque proportional to the sum of gas and radiation pressure. These two assumptions give extremely different predictions.

The second family of models is not strongly influenced by radiation pressure although the radiation pressure dominates in the innermost parts (e.g. Clarke 1988). Models are thermally stable in the inner parts (see Piran 1978) and are not expected to develop any extended optically thin regions which would be a natural site for $x$-ray emission.

The models from the first family, however, are most probably strongly stratified as the unstratified models are known to be thermally unstable (see Sec. 5.2 and 5.3). Some parts of the disks are optically thin (in a sense of effective optical depth), and therefore their emission is predominantly in the x-ray band.

\subsection{OPtICALLY THICK EMISSION FROM UNSTRATIFIED DISKS}

The spectra of unstratified $\alpha$ disks were calculated already by Shakura and Sunyaev (1973) using analytic approximations of the effects of electron scattering and Comptonization. More advanced models were subsequently published (e.g. Czerny \& Elvis 1987, Laor \& Netzer 1989, Sun and Malkan 1989). The best models so far (Ross, Fabian and Mineshige 1993) are based on properly solved radiative transfer, i.e. free-free, bound-free and bound-bound opacities as well as Comptonization calculated in the diffusion approximation (Kompaneetz equation) but for a constant density and constant viscous energy generation in the vertical direction. Fully selfconsistent solutions, with viscous energy deposit given locally by $\alpha$ scaling and the hydrostatic equilibrium taken into account are not available yet for regions dominated by radiation pressure (see Riffer et al, this volume).

These more advanced spectra models are more favorable from the observational point of view. Since the spectra extend to higher frequencies lower accretion rates are required to fit high redshift quasars or those few Seyferts where the optically thick emission extends into soft x-ray band. The flattening of the spectra in the uv range also partially closes the gap with the observed slopes (see Sec. 3). Some external irradiation may then reproduce the observed spectral shape if the accretion rate is close to the critical one; such a model was used both to fit the spectrum and to account for the microlensing limit for the size of emitting region in the "Einstein cross', or Q2237+0305 (Czerny, Czerny and Jaroszyński 1993). 
However, these spectra are not fully reliable as long as the possibility of strong stratification within the disk is not studied and eventually incorporated into the model.

\subsection{RADIAL STRATIFICATION}

The vertically averaged $\alpha$ disks equations are known to have two solutions for a given accretion rate. The first is the Skakura-Sunyaev optically thick solution. The second one is the optically thin branch - a hot ion torus. It was discovered by Shapiro, Lightman and Eardley (1976) within a specific context of non-local model (cooling by external photons) but also local considerations give similar branch (e.g. Bjornsson and Svensson 1992, Kusunose and Zdziarski 1993). The two solutions merge for very high accretion rate (Liang and Wandel 1991) and there are no solutions for higher values of accretion rate at a given radius. The details depend on the assumption about the cooling mechanism, i.e. whether external soft photons or synchrotron photons are present in addition to bremstrahlung emission as the number of soft photons cooling the medium through Compton scattering is essential both for the structure of the disk and the emitted spectrum.

Radially stratified solution consists of two parts: optically thick outer part and optically thin inner part. This radius of change $R_{\text {div }}$ from one branch of solutions to another is in principle uniquely determined by the model from the condition of marginal thermal stability of the outer branch: $P_{g a s} / P_{t o t}=0.4$ (Shakura and Sunyaev 1976).

This criterion predicts rather extended ion torus and the $x$-ray luminosity strongly dominating the big bump luminosity. This is not expected for Seyfert galaxies and quasars, and also it might be difficult to reconcile with the mean energy index between optical and $x$-ray band which tends to be steeper for bright quasars.

However, this criterion changes if timescales for the development of instability are considered (Shakura \& Sunyaev 1976) as well as the irradiation of cool part by the hot ion torus is taken into account as the illumination tends to suppress the instability (e.g. Czerny, Czerny and Grindlay 1986). More detailed consideration of stability are needed to improve it so arbitrarily adopted value of $r_{d i v}$ is a reasonable possibility for the time being.

The existence of two temperature ion torus has been questioned as the collective plasma phenomena may result in a strong coupling between the electrons and ions. However, simple numerical experiment of changing the value of the coupling constant indicate that the torus structure adjust to such a change without decreasing the difference of temperature between ions and electrons (Kusunose \& Zdziarski 1993).

\subsection{Vertical STRATIFICATION}

If the $\alpha$ prescription for viscosity is used locally without averaging the disk structure in the vertical direction also solutions with strong vertical stratification can 
be found which is perhaps closely related to the difficulties in solving both the equations for the disk structure and the radiative transfer self-consistently without approximations (see Sec. 5.1).

We study the possibility of such stratification using two slab approximation $(\mathrm{Cz}-$ erny, Collin-Souffrin \& Życki 1994). We assume that an optically thin hot corona is situated on the top of optically thick disk layer. The viscous heat generation both in the cold layer and in the hot corona are described using the same value of $\alpha$. The interaction of the two slabs is described by (i) assuming the hydrostatic equilibrium at the base of the corona (ii) assuming the flux exchange between the two slabs, as in Haart and Maraschi (1992). The two-temperature corona is cooled by Comptonizing the outgoing soft photons. The pressure in the colder slab is described as a sum of gas and radiation pressure.

Such a set of equations parameterized by $\alpha, M$ and $\dot{M}$ give two solutions at a given radius. The fraction of energy generated in a corona is significant in one solution and much smaller in the other one. The first solution has been found by Nakamura \& Osaki (1994) for the gas pressure dominated disk. However, the two solutions merge at a certain radius and no vertical stratification is predicted at larger radii.

The coronal solution region increases with increasing accretion rate; it is smaller than the division point for radial stratification although it increases faster with $\dot{m}$. However, if the criterion for radial stratification is modified (see Sec. 5.2) then for large accretion rate the coronal solution may develop in the outermost parts and continue inwards. For low accretion rate the ion torus will form unavoidably, according to the model.

\subsection{Observational consequences of alpha models}

Since these models allow for strong stratification effects the detailed observational predictions should include the interaction of separate parts of the disk. Such a full program have not been completed yet but partial results are available.

Hot part of radially stratified disks well reproduce the slope of $x$-ray spectrum if synchrotron emission of soft photons is allowed (e.g. Kusunose and Zdziarski 1993). External soft photons would also give the required spectrum but it is difficult for them to penetrate the hot ion torus as its optical depth for scattering is significant (Wandel \& Liang 1991).

The effect of irradiation of the outer cold part of the disk by the inner torus have been calculated by Rokaki, Collin-Souffrin \& Magnan (1993) . Assuming constant value of the division point but variable $x$-ray luminosity the authors could explain the observed optical/uv variability of NGC 5548; however, additional power law ir/uv component was still required to fit the data.

The emission line profiles in $10 \%$ of radio galaxies are successfully fitted if the extension of the cool disk is given by radiation pressure criterion (Eracleous \& Halpern 1993).

The detailed predictions for vertically stratified models are not available yet 
but they are expected to be qualitatively similar to coronal models (see Sec 6.2).

\section{Modified models}

Since the strict justification for the $\alpha$ scaling of viscous torque does not exist and the limits of applicability are unknown a number of different, still more ad hoc, approaches were suggested.

\subsection{IRRADIATED DISKS WITH UNSPECIFIED X-RAY SOURCE}

Unstratified $\alpha$ disk models have to be arbitrarily supplemented with a source of $\mathbf{x}$-ray emission. Detailed calculations of continuum have been made for a compact source of power law radiation above an accretion disk (Ross \& Fabian 1993, Matt, Ross \& Fabian 1993). Iron $K_{\alpha}$ line predictions were made for the same geometry (Matt, Fabian \& Ross 1993, Życki \& Czerny 1993).

\subsection{Coronal solutions}

Hot corona with gravitational energy release (as opposite to corona caused by external irradiation) not constrained to $\alpha$ viscosity was considered e.g. by Liang \& Thompson (1979). Attractive and simple approach was suggested by Haardt \& Maraschi 1991, later followed by Kusunose \& Mineshige (1994) and Haardt \& Matt (1993). Model is parameterized by a fraction $f$ of energy dissipated in a hot corona. The model gives $\mathbf{x}$-ray spectra close to the observed ones in the case of $f$ close to 1 (Haardt \& Maraschi 1991). The disadvantage of the model is that the entire disk is covered by a corona and all uv radiation is strongly comptonized as well. However, the problems disappears if the corona is clumpy.

\subsection{Optically THIN AND OPTICALly THICK BLOBS}

The presence of blobs instead of accretion disk close to the center was suggested by Rees (1987). Although a spherically symmetric cloud distribution does not seem to be consistent with observations a flattened cloud distribution is actually difficult to distinguish from accretion disk if clouds are optically thick (Malkan 1992). Only very detailed modelling (e.g. Sivron \& Tsuruta 1993) may reveal some subtle differences. Optically thin clouds (Ferland, Korrista \& Peterson 1990, Barvainis 1993) seem to be less convincing. Optically thin irradiated plasma can hardly be kept at the required temperature (Malkan 1992) and the Lyman edge is too large (Dumont, Collin-Souffrin, Czerny \& Życki, in preparation).

\section{Future prospects}

Further progress is mostly expected from new observational constraints of parameters crucial for theoretical models. For example, mean density of the disk can, in principle, be determined both from the shape of the reflected $x$-ray component and from the polarization studies in uv (e.g. Webb et al. 1993). The overall geom- 
etry is perhaps most strongly constrained by detailed modelling of the monitored variable sources. Explicit nonstationary models may be needed and such models are available now only for vary special cases. A lot of work, both observational and at a modelling level, has to be done yet.

\section{Acknowledgements}

I would like to thank Suzy Collin-Souffrin, Jean-Pierre Lasota and Piotr Życki for fruitful discussions. This work was supported in part by grant No. 2 P30401004 financed in 1993-1995 by the Polish State Committee for Scientific Research.

\section{References}

Abramowicz, M., A., Czerny, B., Lasota, J.P., \& Szuszkiewicz, E., 1988, Ap. J., 332,646

Antonucci, R., 1992, in Testing the AGN Paradigm, ed. S. Holt, S. Neff \& C.M. Urry, New York, AIP, p. 486

Barthel, P.D., 1992, in Physics of Active Galactic Nuclei, eds. W.J. Duschl \& S.J. Wagner, Springer-Verlag, p. 637

Barvainis, R., 1992, in Testing the AGN Paradigm, ed. S. Holt, S. Neff \& C.M. Urry, New York, AIP, p. 129

Bechtold, J., Green, R. F., Weymann, R. J., Schmidt, H., Easterbrook, F. B., Sherman, R. D., Wahlquist, H. D. \& Heckman, T. M. 1984, ApJ, 281, 76

Björnsson, G. \& Svensson, R., 1992, Ap. J., 394, 500

Clarke, C.J., 1988, MNRAS, 235, 881

Collin-Souffrin, S., 1992, in Physics of Active Galactic Nuclei, eds. W.J. Duschl \& S.J. Wagner, Springer-Verlag, p. 133

Czerny, B., Czerny, M. \& Grindlay, J.E., 1986

Czerny, B., Czerny, M. \& Jaroszyński, M., 1993, submitted to MNRAS

Czerny, B. \& Elvis, M., 1987, Ap. J., 321, 305

Edelson, R. \& Malkan, M.A., 1986, Ap. J., 308, 59

Eracleous, M. \& Halpern, J.P., 1993, Ap. J. Suppl. (in press)

Fabian A.C. George I.M., 1990, in Treves A., Perola G.C., Stella L., eds, Iron Line Diagnostics in X-ray Sources, Springer-Verlag, Berlin, p. 169

Frank, J., King, A.R. \& Raine, D.J., 1985, Accretion power in astrophysics. Cambridge University Press, Cambridge

Haardt, F., \& Maraschi, L., 1991, Ap. J. 380, L51

Haardt, F. \& Matt, G., 1993, MNRAS, 261, 346

Heckman, T., in Testing the AGN Paradigm, ed. S. Holt, S. Neff \& C.M. Urry, New York, AIP, p. 595

Henri, G. \& Pelletier, G., 1991, Ap. J., 383, L7

Kolykhalov, P.L. \& Sunyaev, R.A., 1984, Adv. Space Rev., 3, 249

Kusunose, M. \& Mineshige, S., 1994, Ap. J. (in press) 
Kusunose, M. \& Zdziarski, A.A., 1993, submitted to Ap. J.

Laor, A. \& Netzer, H., 1989, MNRAS, 238, 897

Liang, E.P. \& Thompson, 1979, MNRAS, 189, 421

Liang, E.P. \& Wandel, A., 1991, Ap. J., 376, 746

Lynden-Bell, D., 1969, Nature, 223, 690

Malkan, M.A., 1988, Adv. Space Res., 8, 249

Malkan, M., 1992, in Physics of Active Galactic Nuclei, eds.W.J. Duschl \& S.J. Wagner, Springer-Verlag, p. 109

Matt,G., Fabian, A.C. \& Ross, R.R., 1993, MNRAS, 262, 179

Mushotzky, R.F, Done, C. \& Pounds, K.A., 1993, Ann. Rev. Astron. Ap. ???

Neugebauer, G., Green, R.F., Matthews, K., Schmidt, M., Soifer, B.T., \& Bennet, J., 1987, Ap. J. Suppl., 63, 515

Novikov, I. \& Thorne, K.S., 1973, in Black Holes, eds. C DeWitt \& B. deWitt, New York, Gordon \& Breach

O'Brien, P.T., Gondhalekar, P.M. \& Wilson, R., 1988, MNRAS, 233, 801

Nakamura, K. \& Osaki, Y., 1994, PASJ (in press)

Piran, T., 1978, Ap. J., 221, 652

Pringle, J.E., 1981, Ann. Rev. Astron. Ap., 19, 137

Rees, M.J., 1984, Ann. Rev. Astron. Ap., 22, 471

Rees, M.J., 1987, MNRAS, 228, 47P

Reimers, D. et al. 1992, Nature, 360, 561

Rokaki, E., Collin-Souffrin, S. \& Magnan, C., 1993, A\&A, 272, 8

Ross, R.R. \& Fabian, A.C., 1993, MNRAS, 261, 74

Ross, R.R., Fabian, A.C. \& Mineshige, S., 1993, MNRAS, 258, 189

Salpeter, E.E., 1964, Ap. J., 140, 796

Sanders, D.B., Phinney, E.S., Neugebauer, G., Soifer, B.T., Mathews, K., Green, R.F., 1989, Ap. J., 347, 29

Schlosman, I., Begelman, M.C. \& Frank, J., 1990, Nature, 345, 679

Shakura, N.I. \& Sunyaev, R.A., 1973, A\&A, 24, 337

Shakura, N.I. \& Sunyaev, R.A., 1976, MNRAS, 175, 613

Shapiro, S.L., Lightman, A.P. \& Eardley, D.M., 1976, Ap. J., 204, 187

Shields, G.A., 1978, Nature, 272, 706

Sivron, R. \& Tsuruta, S., 1993, Ap. J., 402, 420

Sun, W.-H. \& Malkan, M.A., 1989, Ap. J., 346, 68

Terlevich, R., Tenorio-Tagle, G., Franco, J. \& Melnick, J., 1992, MNRAS, 255, 713

Walter, R. \& Fink, H.H., 1993, A\&A, 274, 105

Wandel, A. \& Liang, E.P., 1991, Ap. J., 380, 84

Wilkes, B.J. \& Elvis, M., 1987, Ap. J., 323, 243

Zeldovich,Ya.B. \& Novikov, I.D., 1964, Dokl. Acad. Nauk SSSR, 158, 811

Życki, P.T. \& Czerny, B., 1993, MNRAS (in press) 